\title{
Inhibition of the Rac1-WAVE2-Arp2/3 signaling pathway promotes radiosensitivity via downregulation of cofilin-1 in U251 human glioma cells
}

\author{
TAO ZHOU ${ }^{1}$, CHEN-HAN WANG $^{1}$, HUA YAN $^{1}$, RUI ZHANG ${ }^{1}$, \\ JIN-BING ZHAO ${ }^{1}$, CHUN-FA QIAN ${ }^{1}$, HONG XIAO $^{2}$ and HONG-YI LIU ${ }^{1}$ \\ ${ }^{1}$ Department of Neurosurgery; ${ }^{2}$ Neuropsychiatric Institute, Nanjing Medical University, \\ Affiliated Nanjing Brain Hospital, Nanjing, Jiangsu 210029, P.R. China
}

Received May 14, 2015; Accepted March 16, 2016

DOI: $10.3892 / \mathrm{mmr} .2016 .5088$

\begin{abstract}
The Ras-related C3 botulinum toxin substrate 1 (Rac1)-WASP-family verprolin-homologous protein-2 (WAVE2)-actin-related protein 2/3 (Arp2/3) signaling pathway has been identified to be involved in cell migration and invasion in various types of cancer cell. Cofilin-1 (CFL-1), which is regulated by the Rac1-WAVE2-Arp2/3 signaling pathway, may promote radioresistance in glioma. Therefore, the present study aimed to investigate the potential role of the Rac1-WAVE2-Arp2/3 signaling pathway in radioresistance in U251 human glioma cells and elucidate its affect on CFL-1 expression. Western blot analysis was performed to evaluate the protein expression of CFL-1. In the present study, Rac1 was inhibited by NSC 23766, WAVE2 was inhibited by transfection with short hairpin (sh)RNA-WAVE2 using Lipofectamine $^{\mathrm{TM}} 2000$ and Arp2/3 was inhibited by CK-666. Cell viability was measured using the 3-(4,5-dimethylthiazol-2-yl)-2,5-diphenyltetrazolium bromide assay, the cell migration ability was examined by a wound-healing assay, and the cell invasion ability was assessed using a Transwell culture chamber system. The results showed that inhibition of the Rac1-WAVE2-Arp2/3 signaling pathway using NSC 23766, shRNA-WAVE2 or CK-666 reduced the cell viability, migration and invasion abilities in U251 human glioma cells, concordant with a reduced expression of CFL-1. Furthermore,
\end{abstract}

Correspondence to: Dr Hong-Yi Liu, Department of Neurosurgery, Nanjing Medical University, Affiliated Nanjing Brain Hospital, 264 Guangzhou Road, Nanjing, Jiangsu 210029, P.R. China E-mail: hyliu18@126.com

Dr Hong Xiao, Neuropsychiatric Institute, Nanjing Medical University, Affiliated Nanjing Brain Hospital, 264 Guangzhou Road, Nanjing, Jiangsu 210029, P.R. China

E-mail: xhnkyy123@163.com

Key words: ras-related C3 botulinum toxin substrate 1, WASP-family verprolin-homologous protein-2, actin-related protein 2/3, cofilin-1, radioresistance, radiosensitivity, glioma, $\mathrm{U} 251$ cells the expression of CFL-1 was significantly increased in radioresistant U251 glioma cells when compared with normal U251 human glioma cells. These findings indicate that inhibition of the Rac1-WAVE2-Arp2/3 signaling pathway may promote radiosensitivity, which may partially result from the downregulation of CFL-1 in U251 human glioma cells.

\section{Introduction}

Glioma is the most common type of malignancy of the central nervous system in adults worldwide (1). Glioma has a very poor prognosis and the natural history of the disease is particularly short, typically $<1$ year (2). High-grade glioma presents a challenge with regard to research. Despite the current treatment regimen of surgery and radiotherapy with concomitant temozolomide administration, median survival is $\sim 1$ year $(3,4)$. The poor response to therapy may be due to the intratumoral heterogeneity of these malignancies at the cellular and molecular levels (5-7). Radiotherapy has been important in prolonging the survival of patients with glioma since the 1970s (8). However, although radiotherapy is widely adopted for glioma treatment, the radioresistance of glioma cells limits its success (9).

Ras-related C3 botulinum toxin substrate 1 (Rac1) is among the most extensively characterized members of the Rho family of small GTPases, which are adhesion- and growth-factor activated molecular switches that are important in tumor development and progression $(10,11)$. In addition, Rac1 has been identified to contribute to radioresistance in head and neck squamous cell carcinomas (12). WASP-family verprolin-homologous protein-2 (WAVE2) is a member of the family of Wiskott-Aldrich Syndrome protein (WASp)/WAVE proteins, which is activated at the plasma membrane by Rac1 and uses the actin-related protein 2/3 (Arp2/3) complex to generate a dendritic array of branched filaments responsible for the extension of lamellipodia $(13,14)$. WAVE2 is strongly associated with migration of a range of tumor cells, and is implicated in tumor cell invasion and metastasis via regulating the Arp2/3 complex (15). In addition, activation of Rac results in dephosphorylation of cofilin (16), and the Arp2/3 complex serves as a key upstream factor for the recruitment of 
modulators of lamellipodia formation, such as capping protein or cofilin (17). A previous study demonstrated that blocking the Rac1-WAVE2-Arp2/3 signaling pathway in carcinoma cells results in marked suppression of cell migration and invasion (18).

Cofilin-1 (CFL-1) is a non-muscle isoform of the actin depolymerizing factor/cofilin protein family, which accelerates actin dynamics in vitro and in vivo $(19,20)$. The actin-associated protein, cofilin binds and severs actin filaments (21). In addition, dysfunction of CFL-1 is essential for cell viability and migration $(22,23)$. A previous study revealed that CFL-1 was significantly upregulated in radioresistant astrocytomas (24) and it is potentially involved in decreasing radiosensitivity in U251 cells (25). Thus, the present study proposed that the Rac1-WAVE-Arp2/3 signaling pathway may be involved in the radioresistant phenotype, via CFL-1, and may present as a novel therapeutic target for glioma.

In the present study, U251 cells, a human glioma cell line, were selected as an in vitro model to investigate whether the Rac1-WAVE-Arp2/3 signaling pathway is involved in radioresistance, and to elucidate its regulatory role in CLF-1 expression.

\section{Materials and methods}

Cell culture. U251 human glioma cells were obtained from Nanjing KeyGen Biotech Co. Ltd. (Nanjing, China). Radioresistant U251 (RR-U251) human glioma cells were established according to a previously described method (25). U251 and RR-U251 cells were cultured in Gibco Dulbecco's modified Eagle's medium (DMEM; Thermo Fisher Scientific, Inc., Waltham, MA, USA) supplemented with Gibco $10 \%$ fetal bovine serum (FBS; Thermo Fisher Scientific, Inc.), $100 \mathrm{U} / \mathrm{ml}$ penicillin and $100 \mu \mathrm{g} / \mathrm{ml}$ streptomycin (both Thermo Fisher Scientific, Inc.) in a $37^{\circ} \mathrm{C}$ humidified incubator with $5 \% \mathrm{CO}_{2}$.

Transfection. The short hairpin (sh)RNA-WAVE2 plasmid was synthesized by Santa Cruz Biotechnology, Inc. (Dallas, TX, USA). U251 cells $\left(1 \times 10^{5} / \mathrm{ml}\right)$ were seeded into 6 -well plates $24 \mathrm{~h}$ prior to transfection and $1 \mathrm{ml}$ DMEM (with serum and antibiotics) was added. Upon reaching $~ 50 \%$ confluence the U251 cells in the 6-well plates were transfected using Lipofectamine ${ }^{\text {TM }} 2000$ (Invitrogen; Thermo Fisher Scientific, Inc.) according to the manufacturer's instructions. The medium was replaced with fresh DMEM containing $10 \%$ FBS 6 h after transfection. Following transfection $(24 \mathrm{~h})$, cell fluorescence was observed under a fluorescence microscope (Axiovert 40 CFL; Zeiss AG, Oberkochen, Germany) and the transfection efficiency was determined, according to the number of fluorescent cells.

Cell viability assay. Cell viability was determined using a 3-(4,5-dimethylthiazol)-2,5-diphenyltetrazolium bromide (MTT) assay (Sigma-Aldrich, St. Louis, MO, USA). Briefly, the normal U251 and RR-U251 cells $\left(1 \times 10^{5} / \mathrm{ml}\right)$ were seeded into separate 96-well plates. After $24 \mathrm{~h}, 50 \mu \mathrm{mol} / 1$ NSC 23766 (Topscience Biotech Co., Ltd., Shanghai, China) or $200 \mu \mathrm{mol} / \mathrm{lCK}-666$ (Sigma-Aldrich) dissolved in $150 \mu \mathrm{l}$ dimethyl sulfoxide (DMSO; Biosharp, Hefei, China) was added to each well for 0.5-4 h. The cells treated with NSC 23766, shRNA-WAVE2, and CK-666, and the untreated cells, were irradiated using a cobalt- 60 source at $0.5 \mathrm{~Gy} / \mathrm{min}$ for $10 \mathrm{~min}$, followed by washing with phosphate-buffered saline (PBS), trypsin digestion (Nanjing Sunshine Biotechnology Co., Ltd., Nanjing, China) to separate the cells from the wells and centrifugation at $37^{\circ} \mathrm{C}$ for $6 \mathrm{~min}$ at $300 \mathrm{x} \mathrm{g}$. The cells were then incubated in fresh DMEM (containing antibiotics) for an additional $48 \mathrm{~h}$ at $37^{\circ} \mathrm{C}$. MTT solution $(20 \mu \mathrm{l})$ was added to each well and the cells were incubated at $37^{\circ} \mathrm{C}$ for $4 \mathrm{~h}$. Aliquots of $150 \mu \mathrm{l}$ DMSO were added into each well to dissolve the formazan following removal of the old medium from the plates. The optical density (OD) of the samples was measured at a wavelength of $570 \mathrm{~nm}$ using a microplate reader (Multiskan Ascent; Thermo Fisher Scientific, Inc.). Cell viability (\%) was calculated using the following equation: Cell viability $(\%)=100 x\left(\mathrm{OD}_{\text {treatment }} / \mathrm{OD}_{\text {control }}\right)$.

Cell migration assay. Cell migration was examined using a wound-healing assay. Normal U251 and RR-U251 cells, treated with NSC 23766, shRNA-WAVE2 and CK-666, and the untreated cells were irradiated, which was followed by a washout. Cells were subsequently seeded into 6-well plates and allowed to reach confluency. The glioma cells were scratched using a $200-\mu 1$ tip pipette to create the wound line. Fresh DMEM was added to each well after the plates were washed twice with PBS. Sequential images were obtained using the Axiovert 40 CFL microscope by capturing photomicrographs at 0 and $24 \mathrm{~h}$ at the same site, and the scratch area was measured using a ruler. The rate of migration was further analyzed using the following equation: Migration ratio $=($ Width $0 \mathrm{~h}-$ Width $24 \mathrm{~h}) /$ Width $0 \mathrm{~h} \times 100 \%$.

Cell invasion assessment. Cell invasion was measured using a Matrigel-coated Transwell chamber kit (Corning Incorporated, Corning, NY, USA). The normal U251 and RR-U251 cells, treated with NSC 23766, shRNA-WAVE2 and CK-666, and the untreated cells were irradiated, which was followed by a washout. The monolayer cells were then suspended in serum-free DMEM at a density of $1 \times 10^{5} / \mathrm{ml}$. Aliquots $(100 \mu \mathrm{l})$ of suspension was seeded into the top chambers. DMEM supplemented with $15 \%$ FBS was added to the lower chamber. Cells were then incubated in a $5 \% \mathrm{CO}_{2}$ atmosphere at $37^{\circ} \mathrm{C}$. Following incubation for $24 \mathrm{~h}$, the top chamber was removed and the cells remaining on the upper surface of the membrane were removed using a medical cotton swab. The invasive cells on the lower surface were fixed with formaldehyde (Dynamic Chemical Industry Co., Ltd., Nanjing, China) and stained with crystal violet (Biosharp) for $10 \mathrm{~min}$. The chambers were photographed in five randomly selected fields (magnification, x40) per well under a Axiovert 40 CFL microscope. All experiments were performed three times.

Western blot analysis. U251 and RR-U251 cells were washed with ice-cold PBS three times and then frozen at $-80^{\circ} \mathrm{C}$ for $\geq 24 \mathrm{~h}$ before lysing the cells using a lysis buffer (Thermo Fisher Scientific, Runcorn, UK). Following centrifugation for $15 \mathrm{~min}$ at $1,000 \mathrm{xg}$, the supernatant was collected. All samples were diluted in loading buffer (Nanjing Sunshine Biotechnology Co., Ltd.) and boiled for $3 \mathrm{~min}$. Thirty micrograms of each protein sample were resolved by $10 \%$ sodium dodecyl sulfate-polyacrylamide gel electrophoresis before being blotted onto a 

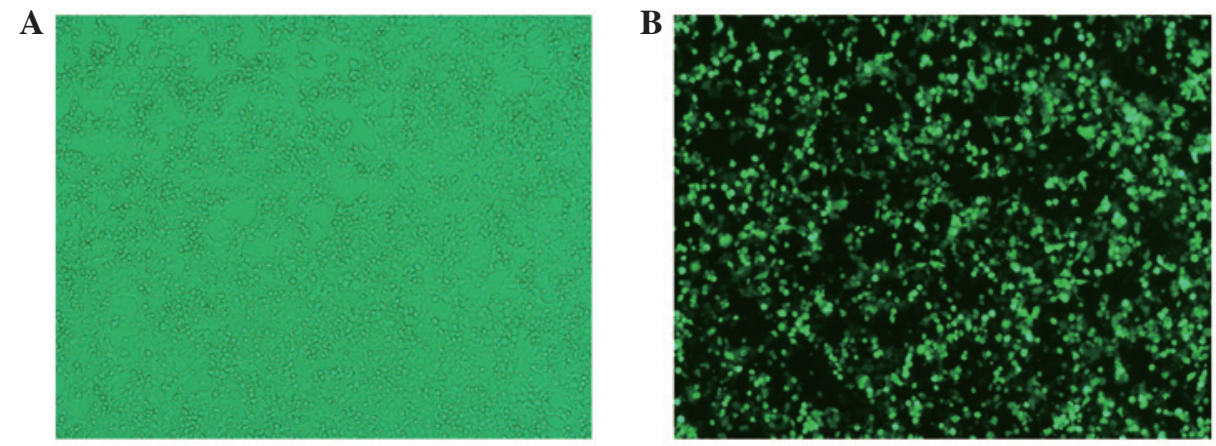

Figure 1. Confirmation of the transfection efficiency. Cells were transfected with a green fluorescent protein-shRNA-WAVE2 using Lipofectamine ${ }^{\mathrm{TM}} 2000$. Following a $24 \mathrm{~h}$ transfection, plates were observed under (A) bright-field and (B) fluorescence microscopy systems (magnification, x20). shRNA, short hairpin RNA; WAVE2, WASP-family verprolin-homologous protein-2.

A

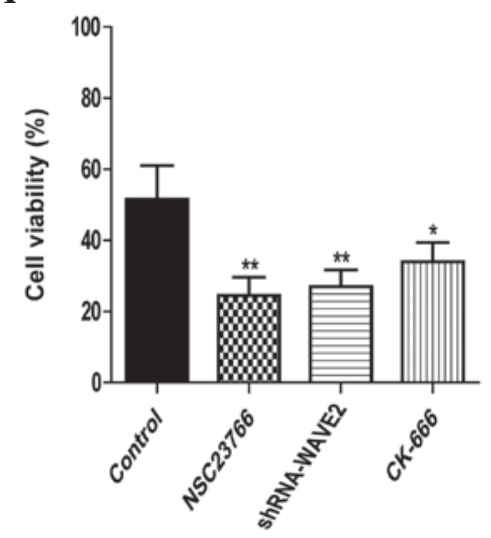

B

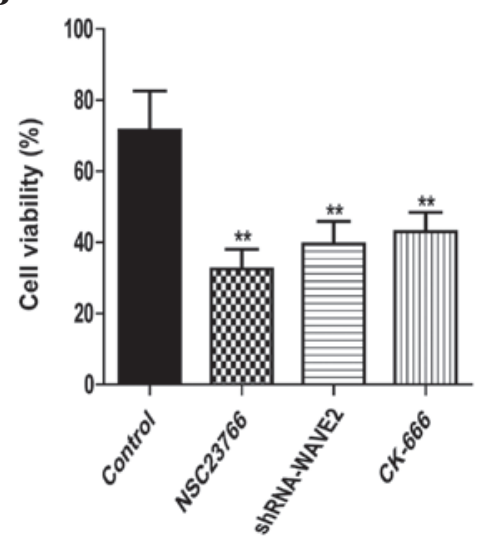

Figure 2. Effects of inhibiting the Ras-related C3 botulinum toxin substrate 1-WAVE2-actin-related protein 2/3 signaling pathway on U251 cell proliferation. (A) Normal U251 cells and (B) radioresistant-U251 cells treated with NSC 23766, shRNA-WAVE2 and CK-666. Untreated cells were exposed to a single dose of cobalt-60. The proliferation ratio was determined using a 3-(4,5-dimethylthiazol-2-yl)-2,5-diphenyltetrazolium bromide assay following $48 \mathrm{~h}$ (n=3). Data are presented as the mean \pm standard deviation. ${ }^{*} \mathrm{P}<0.05,{ }^{* *} \mathrm{P}<0.01$ vs. the control. shRNA, short hairpin RNA; WAVE2, WASP-family verprolin-homologous protein-2.

nitrocellulose membrane (Nanjing Sunshine Biotechnology Co., Ltd.). Following blocking with $5 \%$ non-fat milk, the membranes were incubated overnight at $4^{\circ} \mathrm{C}$ with rabbit anti-CFL-1 polyclonal antibody (1:1,000; cat. no. ab29038; Abcam, Cambridge, MA, USA) and mouse anti-GAPDH monoclonal antibody (1:1,000; cat. no. KC-5G4; Kangchen Biotech Co., Ltd., Zhejiang, China), followed by incubation with horseradish peroxidase-conjugated goat anti-rabbit (1:10,000; cat. no. ZB-2301; Nanjing Shengxing Biotechnology Co., Ltd., Nanjing, China) and goat anti-mouse (1:10,000; cat. no. SN133; Nanjing Sunshine Biotechnology Co., Ltd.) secondary antibodies for $1 \mathrm{~h}$ at room temperature. After washing three times using TBST, the protein bands were detected by enhanced chemiluminescence (ECL; Pierce; Thermo Fisher Scientific, Inc., Shanghai, China). The intensity of protein bands was quantified using Quantity One 4.62 software (Bio-Rad Laboratories, Inc., Hercules, CA, USA).

Statistical analysis. All statistical analyses were conducted using SPSS 19.0 software (IBM SPSS, Armonk, NY, USA). Statistical analyses were performed by means of the Student's $t$-test and the Mann-Whitney $U$ test. Data are presented as the mean \pm standard deviation. $\mathrm{P}<0.05$ was considered to indicate a statistically significant difference.

\section{Results}

Confirmation of transfection efficiency. Transfection of shRNA-WAVE2 duplexes led to a stable exogenous gene expression with $\sim 80-90 \%$ efficiency in U251 cells, as shown by the green fluorescent protein-labeled reporter method (Fig. 1).

Effects of inhibiting the Rac1-WAVE2-Arp2/3 signaling pathway on U251 cell proliferation. The proliferation rates of U251 cells were determined by the MTT assay. The proliferation of normal U251 and RR-U251 cells was significantly downregulated by treatment with NSC $23766(\mathrm{P}=0.005$ and 0.002 , respectively) and CK-666 ( $\mathrm{P}=0.022$ and 0.007 , respectively), and by transfection with shRNA-WAVE2 ( $\mathrm{P}=0.009$ and 0.006 , respectively; Fig. 2).

Inhibition of Rac1-WAVE2-Arp2/3 reduces U251 cell migration ability. The migratory ability of U251 cells was determined using a wound healing assay. Following radiation therapy, the migratory abilities of normal U251 and RR-U251 cells were markedly decreased upon treatment with Racl and Arp2/3 inhibitors, NSC 23766 and CK-666, respectively, or transfection with shRNA-WAVE2, as compared with control cells (Fig. 3). 
A

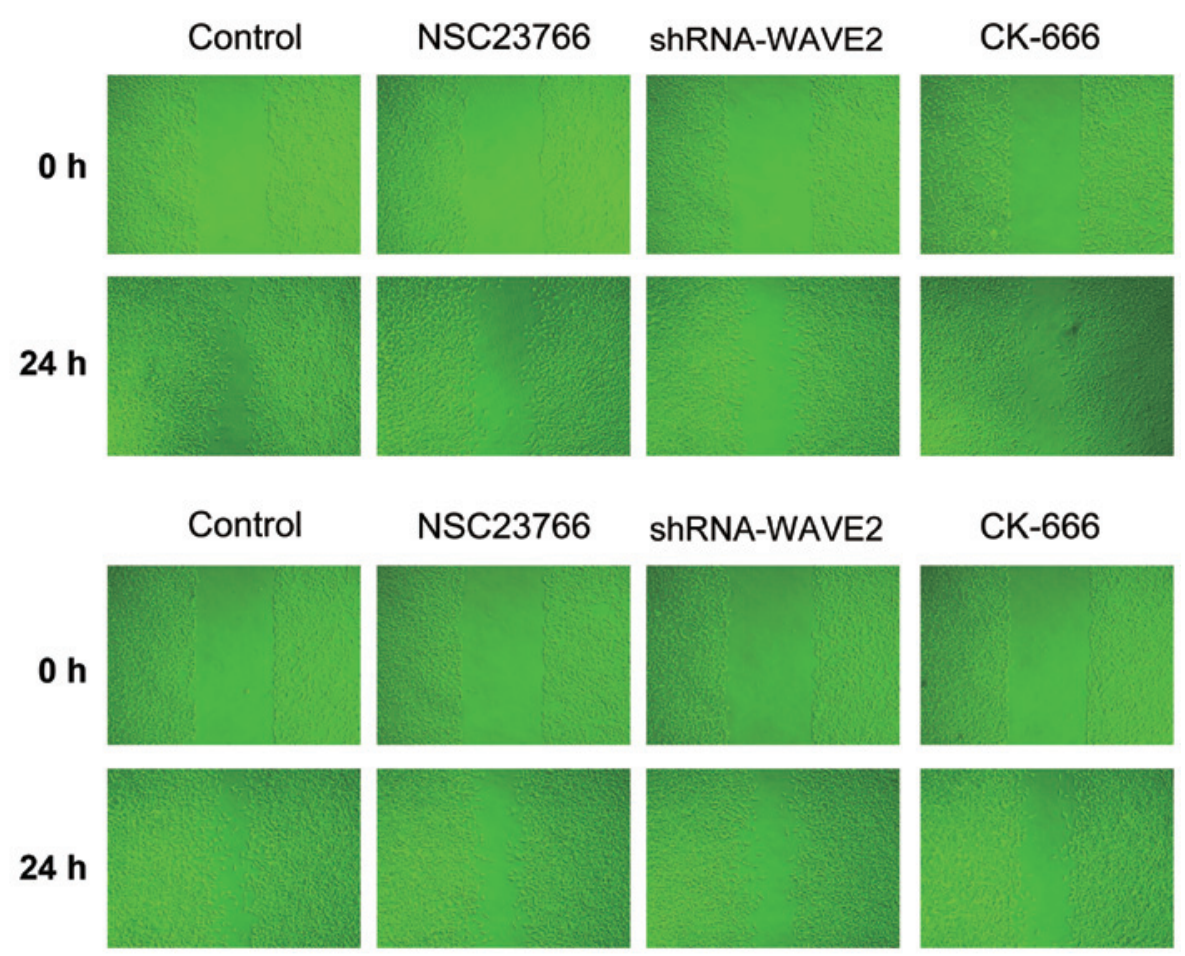

Figure 3. Inhibition of Ras-related C3 botulinum toxin substrate 1-WAVE2-actin-related protein 2/3 reduces U251 cell migration ability. (A) Normal U251 cells and (B) radioresistant-U251 cells treated with NSC 23766, shRNA-WAVE2 and CK-666. Untreated cells were exposed to a single dose of cobalt-60. The cell monolayer cells was scratched using the tip of a 10- $\mu$ l pipette to create a wound line. Images were captured at 0 and $24 \mathrm{~h}$ at identical sites using a fluorescence microscope (magnification, x20). shRNA, short hairpin RNA; WAVE2, WASP-family verprolin-homologous protein-2.

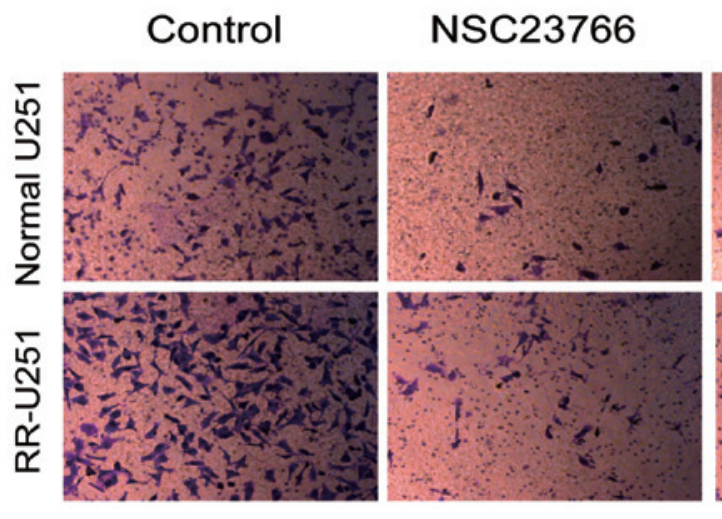

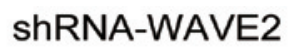

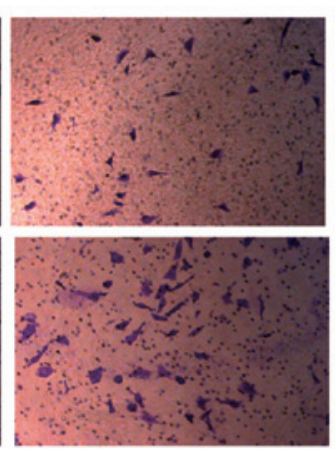

\section{CK-666}

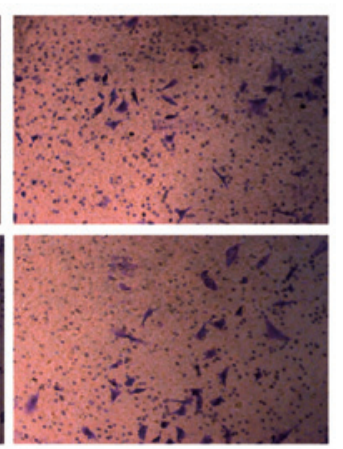

Figure 4. Inhibition of Ras-related C3 botulinum toxin substrate 1-WAVE2-actin-related protein 2/3 impairs U251 cell invasion ability. Normal U251 cells and RR-U251 cells treated with NSC 23766, shRNA-WAVE2, and CK-666. Untreated cells were exposed to a single dose of cobalt-60. Cell invasion ability was evaluated using a Transwell culture chamber system and crystal violet-stained cells were visualized under a microscope (magnification, $\mathrm{x} 40$ ). shRNA, short hairpin RNA; WAVE2, WASP-family verprolin-homologous protein-2; RR, radioresistant.

Inhibition of Racl-WAVE-Arp2/3 impairs U251 cell invasion ability. The role of Racl-WAVE-Arp $2 / 3$ in cell invasion was assessed using the Transwell assay. As presented in Fig. 4, compared with control cells, the invasion potential of U251 cells was inhibited by NSC 23766 and CK-666, and by transfection with shRNA-WAVE2 for Rac1-WAVE2-Arp2/3 in normal U251 and RR-U251 cells. These results indicate that inhibition of Rac1-WAVE-Arp2/3 impairs U251 cell invasion.

Protein expression levels of CFL-1 in U251 and RR-U251 cells. The protein expression of CFL-1 was assessed by western blot and densitometric analyses. As compared with the normal U251 cells, the protein expression level of CFL-1 was significantly elevated in the RR-U251 cells $(\mathrm{P}<0.05$; Fig. 5). Conversely, the protein expression level of CFL-1 was significantly downregulated in normal U251 and RR-U251 cells treated with NSC $23766(\mathrm{P}=0.004$ and $\mathrm{P}<0.001$, respectively) and $\mathrm{CK}-666(\mathrm{P}<0.001$ for both), or transfected with shRNA-WAVE2 ( $\mathrm{P}=0.003$ and 0.002 , respectively), as compared with the control cells (Fig. 6).

\section{Discussion}

In the present study, cell viability, cell migration and cell invasion abilities were observed to be reduced consistently 

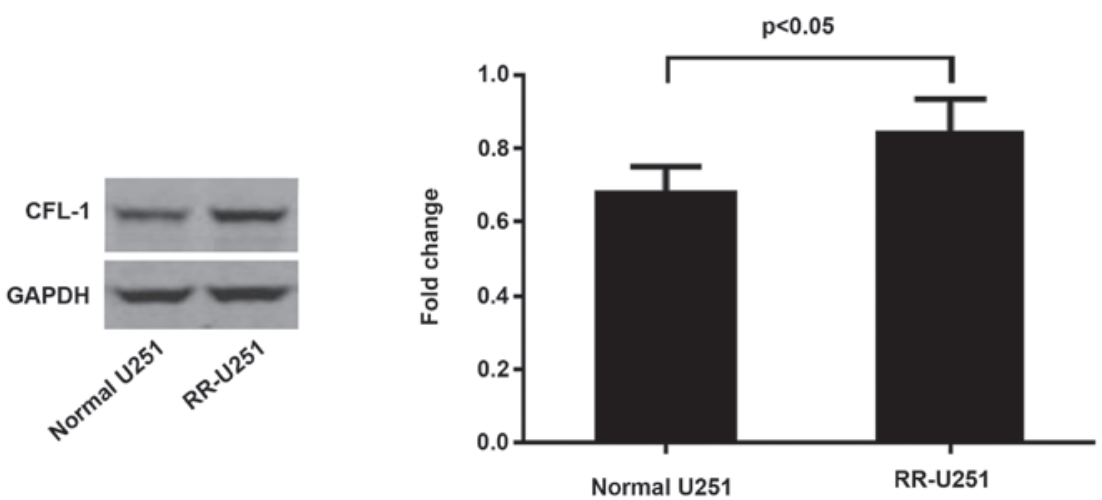

Figure 5. Elevated expression level of CFL-1 protein in RR-U251 cells. Western blot analysis was used to detect protein expression levels of CFL-1 in normal U251 and RR-U251 cells. GAPDH served as the loading control and the relative expression of CFL-1 was determined using densitometry. Data are representative of three independent experiments and are presented as the mean \pm standard deviation. CFL-1, cofilin-1; RR, radioresistant.

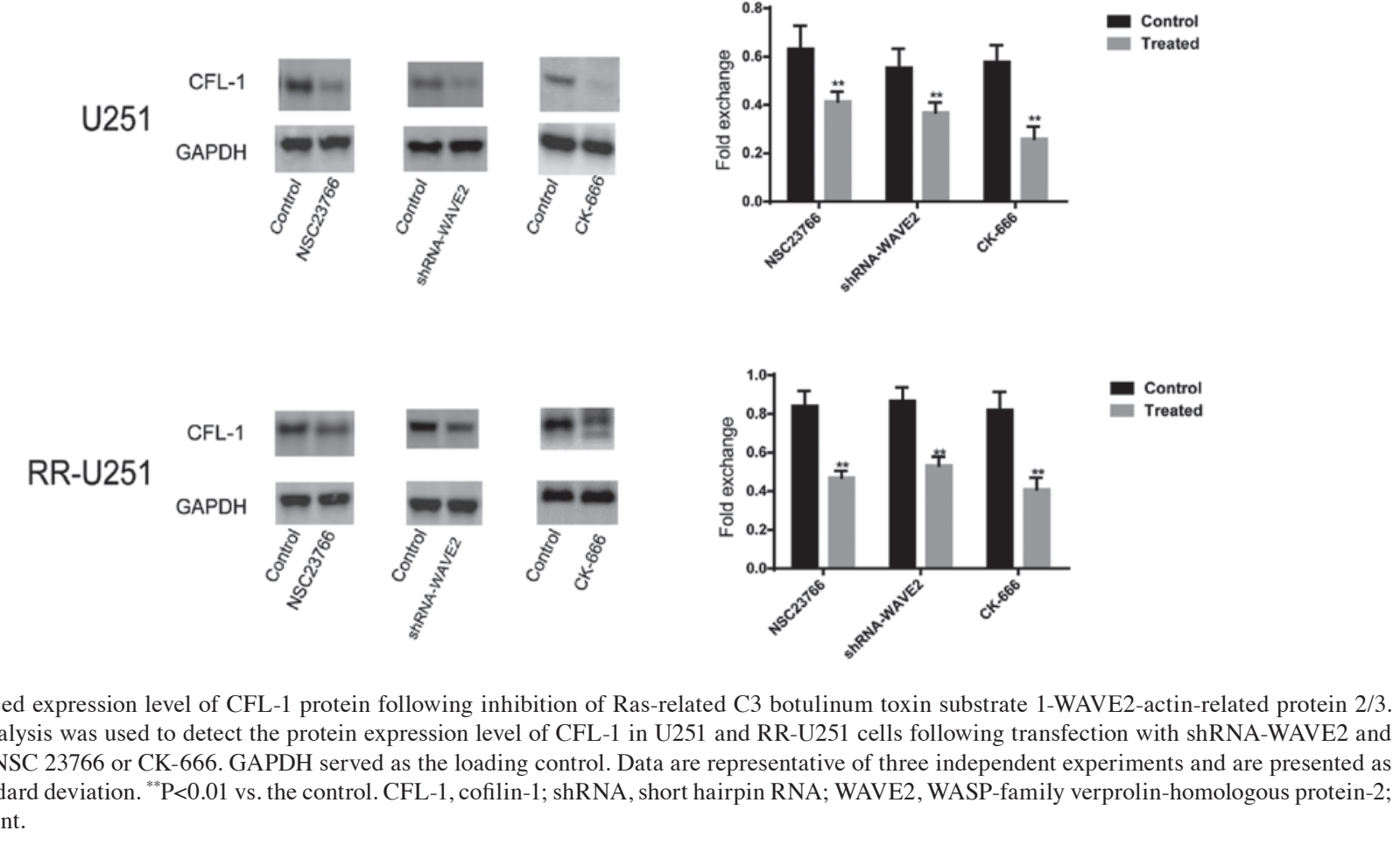

Figure 6. Reduced expression level of CFL-1 protein following inhibition of Ras-related C3 botulinum toxin substrate 1-WAVE2-actin-related protein 2/3 Western blot analysis was used to detect the protein expression level of CFL-1 in U251 and RR-U251 cells following transfection with shRNA-WAVE2 and treatment with NSC 23766 or CK-666. GAPDH served as the loading control. Data are representative of three independent experiments and are presented as the mean \pm standard deviation. ${ }^{* *} \mathrm{P}<0.01$ vs. the control. CFL-1, cofilin-1; shRNA, short hairpin RNA; WAVE2, WASP-family verprolin-homologous protein-2; $\mathrm{RR}$, radioresistant.

with the decreased expression of CFL-1 protein when the Rac1-WAVE2-Arp2/3 signaling pathway was inhibited. These findings indicate that inhibition of the Rac1-WAVE2-Arp2/3 signaling pathway may promote radiosensitivity in U251 human glioma cells, and this regulatory role may involve the downregulation of CFL-1 protein expression, which has been found to contribute to radiosensitivity in human glioma by suppressing tumorigenic properties, including cell viability, cell migration and invasion ability (25).

A previous study reported that CFL-1 potentially contributed to radioresistant astrocytomas and U251 glioma cells $(24,25)$. In addition, it has been shown that Rac1 contributes to radioresistance of head and neck squamous cell carcinomas (12). Rac signaling recruits and activates WAVE, which nucleates branched actin networks via the Arp2/3 complex in tumor development (26). Conversely, Rac is linked to dephosphorylation of cofilin (16). These findings suggest that the Rac1-WAVE-Arp2/3 signaling pathway may be correlated with radiosensitivity and has a marked association with CFL-1 in glioma.

Almost half of all cancer patients receive radiotherapy, which is currently a major treatment option; however, recent reports have suggested that radiotherapy increases the migration and invasion of various cancer cell types, including lung cancer, hepatocellular carcinoma and glioma (27-29). A major factor controlling the metastatic nature of cancer cells is their motility. Alterations in molecular pathways controlling regulation may lead to tumor cell migration and invasion (15). The identification of the signaling pathways of tumor cell metastasis may provide novel diagnostic and therapeutic markers of treatment-resistant cancer (30). Racl activation induces lamellipodia formation via WAVE, which uses the Arp2/3 complex to promote the formation of densely branched filaments (31). Evidence has indicated that Arp2/3 and cofilin act synergisti- 
cally to generate novel actin filaments (32). The present study proposed that the Rac1-WAVE-Arp2/3 signaling pathway is involved in radioresistance in U251 glioma cells exhibiting a high CFL-1 protein expression level.

Metastatic progression of malignant tumors resistant to conventional therapeutic approaches is a challenge in clinical oncology. Despite previous investigation and clinical research, no effective treatment has been established to prevent or combat the metastatic spread of malignant tumors (33). Tumor invasion and metastasis are important factors influencing the poor prognosis and severity of cancer, hence elucidating the mechanism of invasion and metastasis is considered to be a crucial task.

The mechanisms of metastasis include cell detachment, migration, invasion into the surrounding tissue, extravasations, and transplantation followed by formation of tumor emboli (34). It has been reported that inhibition of the Rac1-dependent signaling pathway is an important mechanism underlying cancer metastasis, particularly in cancer cell invasion (35). Cancer cell migration and invasion are closely associated with lamellipodia formation and migration, and the extension of lamellipodia is driven by WASp family proteins, which activate the Arp2/3 complex to catalyze the formation of a branched actin filament array at the leading edge of lamellipodia (36). In addition, cofilin localization is dependent on the mechanism by which cell peripheral actin networks are nucleated and maintained, and its targeting is highly sensitive to Arp $2 / 3$ complex activity and cannot be solely mediated by interaction with actin filaments (37).

Cell migration and invasion is significantly increased in radioresistant glioma cells (21), and dysregulation of certain targets may regulate the signaling pathways of tumor metastasis (38). The identification of CFL-1 as an important downstream effector protein of the Rac1-WAVE2-Arp2/3 signaling pathway, which mediates tumor cell migration and invasion in vitro, highlights the essential role of the Rac1-WAVE2-Arp2/3 signaling pathway in CFL-1 promoting radioresistance in $\mathrm{U} 251$ human glioma cells. Although a direct interaction between CFL-1 and the Rac1-WAVE2-Arp2/3 signaling pathway was not demonstrated in the present study, the results suggested that they may act in concert.

In conclusion, the results of the present study demonstrated that the Rac1-WAVE2-Arp2/3 signaling pathway promotes radioresistance via CFL-1 in U251 human glioma cells in vitro; however, further studies are required to examine the exact molecular mechanism underlying this effect. Additional glioma cell lines should be investigated to confirm the role of CFL-1 and the Rac1-WAVE2-Arp2/3 signaling pathway in glioma. In addition, further studies using rodent models are required to determine the mechanism by which CFL-1 and the Rac1-WAVE2-Arp2/3 signaling pathway contribute to the radioresistance of glioma. The results of the present study may provide a basis for the use of gene therapy in the treatment of patients with glioma.

\section{Acknowledgements}

The present study was supported by grants from the National Natural Science Foundation of China (grant no. NSFC81172390) and the Health Bureau of Nanjing (grant no. ZKX10021).

\section{References}

1. Ford E, Catt S, Chalmers A and Fallowfield L: Systematic review of supportive care needs in patients with primary malignant brain tumors. Neuro Oncol 14: 392-404, 2012.

2. Pace A, Metro G and Fabi A: Supportive care in neurooncology. Curr Opin Oncol 22: 621-626, 2010.

3. Catt S, Chalmers A and Fallowfield L: Psychosocial and supportive-care needs in high-grade glioma. Lancet Oncol 9: 884-891, 2008.

4. Stupp R, Mason WP, van den Bent MJ, Weller M, Fisher B, Taphoorn MJ, Belanger K, Brandes AA, Marosi C, Bogdahn U, et al: Radiotherapy plus concomitant and adjuvant temozolomide for glioblastoma. N Engl J Med 352: 987-996, 2005.

5. Shapiro JR, Yung WK and Shapiro WR: Isolation, karyotype and clonal growth of heterogeneous subpopulations of human malignant gliomas. Cancer Res 41: 2349-2359, 1981

6. Bonavia R, Inda MM, Cavenee WK and Furnari FB: Heterogeneity maintenance in glioblastoma: A social network. Cancer Res 71: 4055-4060, 2011.

7. Sottoriva A, Spiteri I, Piccirillo SG, Touloumis A, Collins VP, Marioni JC, Curtis C, Watts C and Tavaré S: Intratumor heterogeneity in human glioblastoma reflects cancer evolutionary dynamics. Proc Natl Acad Sci USA 110: 4009-4014, 2013.

8. Walker MD, Strike TA and Sheline GE: An analysis of dose-effect relationship in the radiotherapy of malignant gliomas. Int J Radiat Oncol Biol Phys 5: 1725-1731, 1979.

9. Huang L, Li B, Tang S, Guo H, Li W, Huang X, Yan W and Zou F: Mitochondrial KATP channels control glioma radioresistance by regulating ROS-Induced ERK activation. Mol Neurobiol 52: 626-637, 2015.

10. Michaelson D, Abidi W, Guardavaccaro D, Zhou M, Ahearn I, Pagano M and Philips MR: Rac1 accumulates in the nucleus during the G2 phase of the cell cycle and promotes cell division. J Cell Biol 181: 485-496, 2008.

11. Prudnikova TY, Rawat SJ and Chernoff J: Molecular pathways: Targeting the kinase effectors of RHO-family GTPases. Clin Cancer Res 21: 24-29, 2015.

12. Skvortsov S, Dudás J, Eichberger P, Witsch-Baumgartner M, Loeffler-Ragg J, Pritz C, Schartinger VH, Maier H, Hall J, Debbage $\mathrm{P}$, et al: Rac1 as a potential therapeutic target for chemo-radioresistant head and neck squamous cell carcinomas (HNSCC). Br J Cancer 110: 2677-2687, 2014.

13. Machesky LM and Insall RH: Signaling to actin dynamics. J Cell Biol 146: 267-272, 1999.

14. Pollard TD and Borisy GG: Cellular motility driven by assembly and disassembly of actin filaments. Cell 112: 453-465, 2003.

15. Lane J, Martin T, Weeks HP and Jiang WG: Structure and role of WASP and WAVE in Rho GTPase signalling in cancer. Cancer Genomics Proteomics 11: 155-165, 2014.

16. Ma X, Espana-Serrano L, Kim WJ, Thayele PH, Nie Z and Daaka Y: $\beta$ Arrestin1 regulates the guanine nucleotide exchange factor RasGRF2 expression and the small GTPase Rac-mediated formation of membrane protrusion and cell motility. J Biol Chem 289: 13638-13650, 2014.

17. Koestler SA, Steffen A, Nemethova M, Winterhoff M, Luo N, Holleboom JM, Krupp J, Jacob S, Vinzenz M, Schur F, et al: Arp2/3 complex is essential for actin network treadmilling as well as for targeting of capping protein and cofilin. Mol Biol Cell 24: 2861-2875, 2013.

18. Ko HS, Kim JS, Cho SM, Lee HJ, Ahn KS, Kim SH and Lee EO: Urokinase-type plasminogen activator expression and Rac1/WAVE-2/Arp2/3 pathway are blocked by pterostilbene to suppress cell migration and invasion in MDA-MB-231 cells. Bioorg Med Chem Lett 24: 1176-1179, 2014.

19. Bamburg JR: Proteins of the ADF/cofilin family: Essential regulators of actin dynamics. Annu Rev Cell Dev Biol 15: 185-230, 1999.

20. Carlier MF, Ressad F and Pantaloni D: Control of actin dynamics in cell motility. Role of ADF/cofilin. J Biol Chem 274: 33827-33830, 1999.

21. Maciver SK and Hussey PJ: The ADF/cofilin family: Actin-remodeling proteins. Genome Biol 3: reviews3007, 2002.

22. Moriyama K and Yahara I: The actin-severing activity of cofilin is exerted by the interplay of three distinct sites on cofilin and essential for cell viability. Biochem J 365: 147-155, 2002.

23. Gurniak CB, Perlas E and Witke W: The actin depolymerizing factor n-cofilin is essential for neural tube morphogenesis and neural crest cell migration. Dev Biol 278: 231-241, 2005. 
24. Yan H, Yang K, Xiao H, Zou YJ, Zhang WB and Liu HY: Over-expression of cofilin-1 and phosphoglycerate kinase 1 in astrocytomas involved in pathogenesis of radioresistance. Cns Neurosci Ther 18: 729-736, 2012.

25. Du HQ, Chen L, Wang Y, Wang LJ, Yan H, Liu HY and Xiao H: Increasing radiosensitivity with the downregulation of cofilin-1 in U251 human glioma cells. Mol Med Rep 11: 3354-3360, 2015.

26. Dang I, Gorelik R, Sousa-Blin C, Derivery E, Guérin C, Linkner J, Nemethova M, Dumortier JG, Giger FA, Chipysheva TA, et al: Inhibitory signalling to the Arp2/3 complex steers cell migration. Nature 503: 281-284, 2013.

27. Ho JN, Kang GY, Lee SS, Kim J, Bae IH, Hwang SG and Um HD Bcl-XL and STAT3 mediate malignant actions of gamma-irradiation in lung cancer cells. Cancer Sci 101: 1417-1423, 2010.

28. Cheng JC, Chou CH, Kuo ML and Hsieh CY: Radiation-enhanced hepatocellular carcinoma cell invasion with MMP-9 expression through PI3K/Akt/NF-kappaB signal transduction pathway. Oncogene 25: 7009-7018, 2006.

29. Park CM, Park MJ, Kwak HJ, Lee HC, Kim MS, Lee SH, Park IC Rhee $\mathrm{CH}$ and Hong SI: Ionizing radiation enhances matrix metalloproteinase-2 secretion and invasion of glioma cells through Src/epidermal growth factor receptor-mediated p38/Akt and phosphatidylinositol 3-kinase/Akt signaling pathways. Cancer Res 66: 8511-8519, 2006.

30. Wang W, Eddy R and Condeelis J: The cofilin pathway in breast cancer invasion and metastasis. Nat Rev Cancer 7: 429-440, 2007.
31. Le Clainche C, Schlaepfer D, Ferrari A, Klingauf M, Grohmanova K, Veligodskiy A, Didry D, Le D, Egile C, Carlier MF and Kroschewski R: IQGAP1 stimulates actin assembly through the N-WASP-Arp2/3 pathway. J Biol Chem 282: 426-435, 2007.

32. DesMarais V, Macaluso F, Condeelis J and Bailly M: Synergistic interaction between the Arp2/3 complex and cofilin drives stimulated lamellipod extension. J Cell Sci 117: 3499-3510, 2004.

33. Arnold CR, Abdelmoez A, Thurner G, Debbage P, Lukas P, Skvortsov S and Skvortsova II: Rac1 as a multifunctional therapeutic target to prevent and combat cancer metastasis. Oncoscience 1: 513-521, 2014.

34. Gupta GP and Massagué J: Cancer metastasis: Building a framework. Cell 127: 679-695, 2006.

35. Wang C,Lu S, Jiang J,Jia X, Dong X and Bu P: Hsa-microRNA-101 suppresses migration and invasion by targeting Racl in thyroid cancer cells. Oncol Lett 8: 1815-1821, 2014.

36. Lorenz M, Yamaguchi H, Wang Y, Singer RH and Condeelis J: Imaging sites of $\mathrm{N}$-wasp activity in lamellipodia and invadopodia of carcinoma cells. Curr Biol 14: 697-703, 2004.

37. Bernstein BW and Bamburg JR: ADF/cofilin: A functional node in cell biology. Trends Cell Biol 20: 187-195, 2010.

38. Sreekumar R, Sayan BS, Mirnezami AH and Sayan AE: MicroRNA control of invasion and metastasis pathways. Front Genet 2: 58, 2011. 